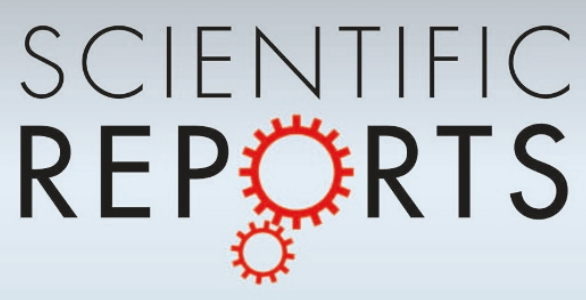

\title{
$-$ High yield production and purification of few layer graphene by Gum Arabic assisted physical sonication \\ SUBJECT AREAS: \\ SYNTHESIS OF \\ GRAPHENE
}

SYNTHESIS AND PROCESSING

TWO-DIMENSIONAL MATERIALS

NANOPARTICLES

Received

28 December 2012

Accepted

13 February 2013

Published

12 March 2013

Correspondence and requests for materials should be addressed to

A.Y. (aipingyu@ uwaterloo.ca)

\author{
Victor Chabot, Brian Kim, Brent Sloper, Costas Tzoganakis \& Aiping Yu
}

Department of Chemical Engineering, Waterloo Institute for Nanotechnology, Waterloo Institute for Sustainable Energy, University of Waterloo, Canada.

Exploiting the emulsification properties of low cost, environmentally safe Gum Arabic we demonstrate a high yield process to produce a few layer graphene with a low defect ratio, maintaining the pristine graphite structure. In addition, we demonstrate the need for and efficacy of an acid hydrolysis treatment to remove the polymer residues to produce $100 \%$ pure graphene. The scalable process gives yield of up to 5 wt $\%$ graphene based on $10 \mathrm{~g}$ starting graphite. The graphene product is compared with reduced graphene oxide produced through Hummer's method using UV-visible spectroscopy, SEM, TEM, and Raman spectroscopy. The two graphene materials show significant difference in these characterizations. Further, the film fabricated from this graphene exhibits 20 times higher electrical conductivity than that of the reduced graphene oxide. Sonication processing of graphite with environmentally approved biopolymers such as Gum Arabic opens up a scalable avenue for production of cheap graphene.

ingle layer and few layer graphenes exhibit a two dimensional carbon lattice structure with outstanding properties including high surface area as well as strong electronic, mechanical, thermal and chemical properties $^{1,2}$. These properties have created considerable scientific interest, driving the pursuit of a scalable production method for high quality graphene sheets.

The first discovery of graphene was carried out by scotch taping peeling, although this approach can obtain pure graphene sheets, the process is not economical and impossible for mass production. Production of few layer graphene by chemical vapor deposition is promising ${ }^{1,3,4}$, however materials can exhibit a low purity mixture of amorphous carbon and the method does not address applications of graphene that require macroscale deployment and high yield. Currently, the most prominent technique for the scalable production of few layer graphene is the chemical reduction or thermal treatment of graphene oxide $(\mathrm{GO})$ from Hummer's method ${ }^{5-10}$. However, the oxidization process also exposes a large number of structural defects within the graphene sheets that compromise some of the properties and the unique morphology of the pristine two dimensional hexagonal carbon lattices ${ }^{10-13}$. Further, the multistep process, the concentrated acids used in oxidization and the harsh chemicals needed to reduce GO increase the economic, safety and environmental costs involved in large scale production.

The drawbacks of the GO process have led to pursuit of easily scaled processes to produce graphene with low basal plane and edge defects. It has previously been shown that sonication of graphite with solvent or surfactant can produce graphene flakes with low defect concentration. Exfoliation through organic solvents containing aromatic donors such as ortho-dichlorobenzene, n-methylpyrrolidone and benzylamine have shown stable dispersions up to $1 \mathrm{mg} / \mathrm{mL}$ through extended low power bath sonication, but these solvents are expensive and require special handling ${ }^{14-17}$. Surfactant based methods are also being investigated for large scale production, but are currently limited by low concentrations of up to $0.05 \mathrm{mg} / \mathrm{mL}^{18,19}$. Longer sonication periods (400 hours) were shown to increase exfoliation concentration up to $0.3 \mathrm{mg} / \mathrm{ml}$ using sodium cholate ${ }^{20}$. However, some surfactants exhibit bioaccumulation and are capable of adsorbing to proteins, disrupting enzyme function and causing organ damage $^{21-23}$. The necessary waste water treatments required to limit mammalian exposure could add cost to the exfoliation process, reducing value.

In this work, we demonstrate Gum Arabic as a green alternative for the exfoliation of graphite to produce graphene in water. Gum Arabic (GA) is a slightly acidic biopolymer which offers strong emulsification properties, high solubility in water, low viscosity and solution stability over a large $\mathrm{pH}$ range $\mathrm{e}^{24,25}$. GA has been used to debundle SWNT in solution, forming stable ink dispersions of individual SWNTs and illustrating its ability to disperse carbon particles ${ }^{26}$. The major component of the structure is composed of a highly branched 
polysaccharide $\left(\mathrm{MW}_{\mathrm{n}}=250 \mathrm{kDa}\right)^{27}$. The secondary component is a surface active glycoprotein which physically adsorbs through steric repulsion, contributing to the materials strong emulsifying property $^{27,28}$. Benefits of Gum Arabic include its low cost, established safety and low environmental risks illustrated through extensive use in food production, such as coca-cola.

Utilizing GA, we have achieved dispersions containing 0.5$0.6 \mathrm{mg} / \mathrm{ml}$ of few layer graphene in $\mathrm{DI}-\mathrm{H}_{2} \mathrm{O}$ through mild sonication. After acid hydrolysis treatment and freeze-dryer, we are able to acquire pure graphene powder. The graphene is almost defect free, having much higher electrical conductivity compared to that from reduced GO ( $-\mathrm{GO})$. The solution based approach is scalable, requires minimum capital investment on the chemicals and equipment, and enables the supply of affordable graphene products to the market. And this is the first time report of production of pure graphene by sonication method.

\section{Results}

As summarized in figure 1(a), graphene synthesis was performed by mixing GA with graphite and subjecting the solution to low power sonication. After exfoliation, the poorly exfoliated graphite solids were then allowed to fully settle before collecting the stably dispersed solution of graphene. After mild centrifuge and filtration, the GA coated graphene was collected as powder and denoted as GA-G. The powder GA-G was then subjected to acid hydrolysis treatment to get rid of the residue GA to achieve pure graphene.

The resulting black pure graphene powder (denoted as $G$ ), seen in figure 1(b), differs visually from the shiny metallic grey of the starting graphite. To better illustrate the dispersion quality figure 1(c) illustrates the captured GA-G solution. The clear seen path of the laser and lack of precipitate on solution bottom depicts a stable colloid dispersion, even after 48 hours. The scattering of the light off the stable particles creates what is known as a Tyndall effect. For comparison, figure $1(\mathrm{~d})$ depicts that without the presence of GA; suspension of the $G$ powder was unstable and became saturated at very low concentrations. The control sample in figure 1(e) contains dissolved GA at a high concentration of $20 \mathrm{mg} / \mathrm{mL}$ and Tyndall effect is barely seen, indicating its presence does not greatly affect the scattering of graphene in figures 1 (c) and $1(\mathrm{~d})$.

For most applications, removal of the residual GA biopolymer is crucial since the residential GA will hamper the properties of graphene. For graphene materials prepared through surfactant exfoliation methodology a significant amount of dispersant residue may remain anchored to the graphene surface even after cleaning ${ }^{20,29}$. Thus, most of the graphene reported by using surfactant is actually mixture of the surfactant and graphene and un-pure. After several exploration of the technique to remove GA, we found that the filtration combined with acid hydrolysis is the best solution to totally removal of GA. Thermo-gravimetric analysis (TGA) is used to detect the amount of GA remaining in the derived GA-G powders. Figure 2(a) gives the TGA and differential (DTG) curves of the GA-G powder after filtration. As illustrated in Figure 2(a), there are two stages of degradation separated by a transition region. The first stage which occurs between $200-400^{\circ} \mathrm{C}$ is in agreement with the bulk degradation temperatures of GA found in literature 27. The remaining GA residue burns away slowly as the temperature increases to $550^{\circ} \mathrm{C}$ suggesting approximately $23 \%$ GA mass left in the dried GA-G powder. Finally, between $550^{\circ} \mathrm{C}$ and $750^{\circ} \mathrm{C}$ the graphitic carbon burns off accounting for the second burn stage.

In order to give an accurate sense of material quality it is important to remove the remaining $23 \% \mathrm{GA}$ in the graphene powder. Figure 2(b) displays the TGA curve of the purified graphene after acid hydrolysis and freeze drying. From the DTG, it is only graphitic peak seen and TG shows the residue almost zero, which confirms the successful GA removal and the stability of the graphitic structure up to $750^{\circ} \mathrm{C}$. Thus the graphene produced by our process is totally free of the GA, and is readily for any desired application.

To further compare the graphene prepared by sonication assisted approach and the r-GO produced by reduction and Hummer's method. We had preformed UV-Vis spectra on the samples. Figure 3(a) compares the UV-vis absorption of the GA-G, G, GA and r-GO. For all the solutions containing graphene, there is a peak centered at $268 \mathrm{~nm}$, and a nearly constant absorbance above $600 \mathrm{~nm}$. The characteristic peak from GA reveals minimal absorbance at $268 \mathrm{~nm}$, but a strong absorbance below $225 \mathrm{~nm}$. This demonstrates (a)
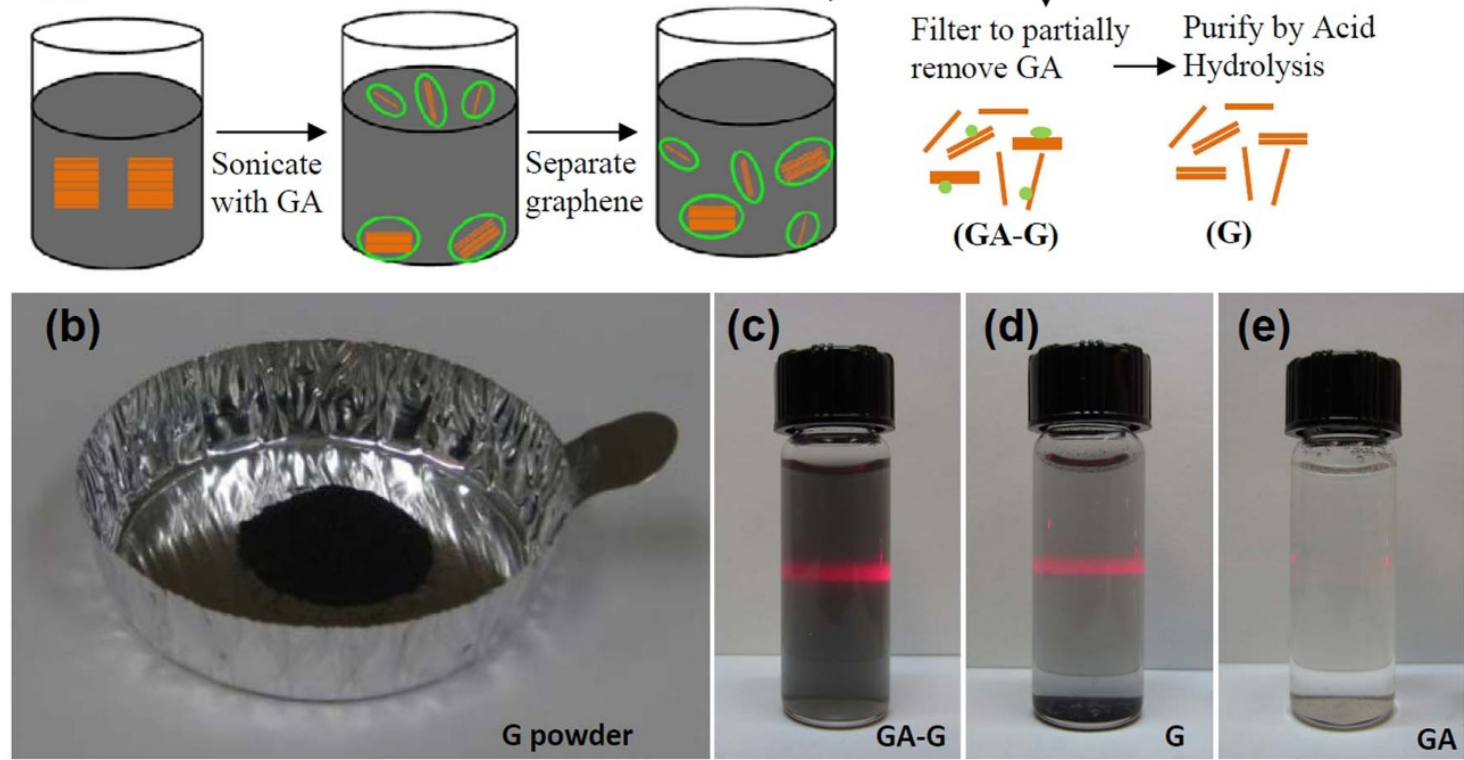

Figure $1 \mid$ (a) Schematic for obtaining a few layer graphene. (b) Pure graphene powder, extracted from 1 L solution. Dispersion quality at diluted concentration $(0.02 \mathrm{mg} / \mathrm{ml})$ for (c) as extracted GA-G, (d) re-dispersion G and (e) pure GA dissolved in solution. 

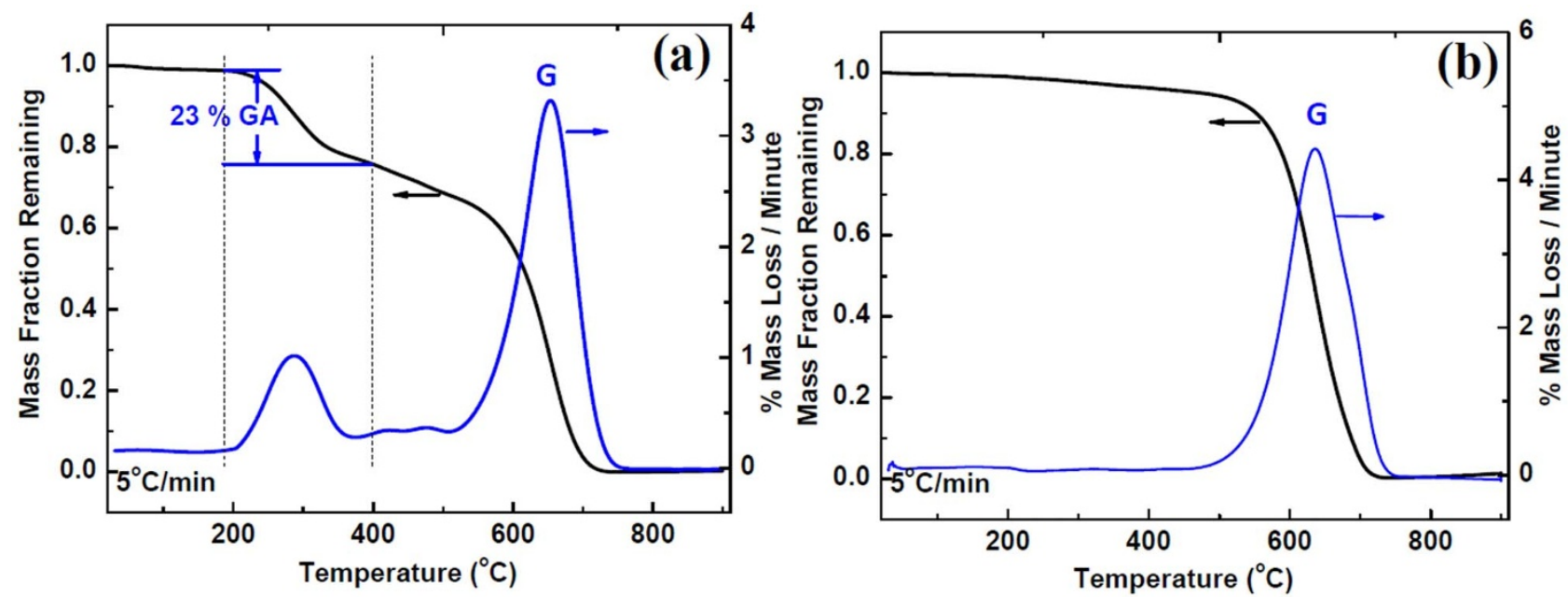

Figure $2 \mid$ Thermo-gravimetric analysis (TGA, black) and the differential temperature curves (DTG,blue) of (a) GA-G and (b) G samples using a temperature ramp of $5^{\circ} \mathrm{C} / \mathrm{min}$.

that both graphene from different production technology has characteristic peak at UV range.

Figure 3(b) demonstrates the calculation of band-gap of different graphene materials based on the UV-spectra. To calculate the optical bandgap, $\mathrm{E}_{\mathrm{g}}$, Tauc's equation was used ${ }^{30}$ :

$$
\omega^{2} \varepsilon^{\prime}=\left(h \omega-E_{g}\right)^{2}
$$

where $\varepsilon^{\prime}$ is the complex part of the dielectric function which is proportional to the optical absorbance according to Tauc ${ }^{31} . \omega=2 \pi / \lambda$, is the angular frequency of the incident radiation. According to the technique, the plot of $\varepsilon^{\prime 0.5} / \lambda$ versus $1 / \lambda$ is a straight line and the intersection point with the $\mathrm{x}$-axis is $1 / \lambda_{\mathrm{g}}\left(\lambda_{\mathrm{g}}\right.$ is the gap wavelength) $)^{32-34}$. The optical band gap is then calculated based on $E g=$ $h c / \lambda_{g}$. The bandgap curve is shown in figure $3(\mathrm{~b})$ and from the intercept we can determine that the bandgaps are $1.46 \mathrm{eV}$ and $1.62 \mathrm{eV}$, for $\mathrm{G}$ and $\mathrm{r}-\mathrm{GO}$ respectively. The slightly lower bandgap might suggest high retention of graphitic character due to the abundance of delocalized $\mathrm{sp}^{2}$ hybridized carbons. Meanwhile, the $\mathrm{r}-\mathrm{GO}$ is an attempt to restore the integrity and conductivity of the graphene basal plane. Based on the band-gap data, we can predict that after removal of residual GA the conductivity of $\mathrm{G}$ will be higher than that of r-GO.

To further quantify the graphene character a series dilution is performed and analyzed for the graphenes' baseline absorbance at $660 \mathrm{~nm}$. Figures 3(c) and 3(d) depict the corrected graphene concentration within the $\mathrm{G}$ and the $\mathrm{r}-\mathrm{GO}$ samples, respectively. The extinction coefficients shown in Table 1 are determined by extrapolating and applying beers law:

$$
\mathrm{A} / \mathrm{L}=\varepsilon C
$$

Where transmission length (L) is constant and extinction coefficient $(\varepsilon)$ is constant for a specific material and wavelength.

As can be seen from the table 1, the extinction coefficient for $\mathrm{G}$ is calculated to be $5422 \mathrm{ml}^{*} \mathrm{mg}^{-1 *} \mathrm{~m}^{-1}$. This is much higher than that for $\mathrm{r}-\mathrm{GO}$ which exhibits a value of $2813 \mathrm{ml}^{*} \mathrm{mg}^{-1 *} \mathrm{~m}^{-1}$ (Figure 3(d)). $\mathrm{G}$ is more comparable to the $6600 \mathrm{ml}^{*} \mathrm{mg}^{-1 *} \mathrm{~m}^{-1}$ seen by Lotya et al for higher concentration dispersions achieved by long sonication time in $\mathrm{NMP}^{20}$. The extinction coefficient is assumed to be a characteristic material property, while the distinct values probably represent the different layer and surface properties of the products ${ }^{35,36}$.
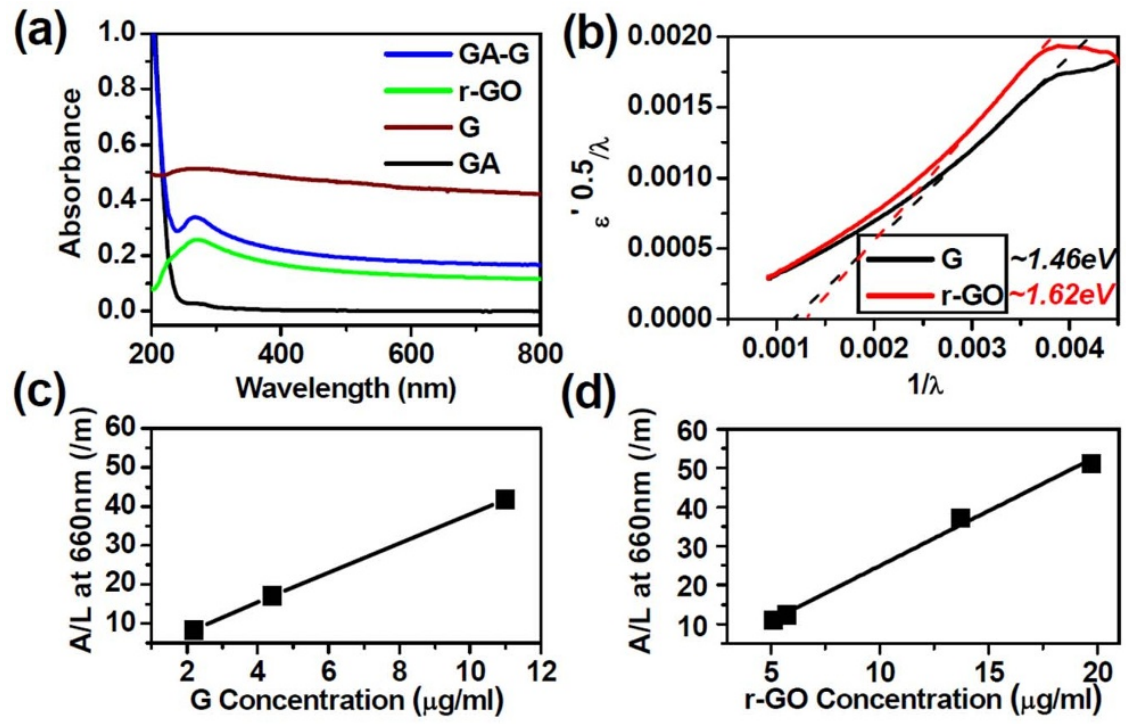

(d)

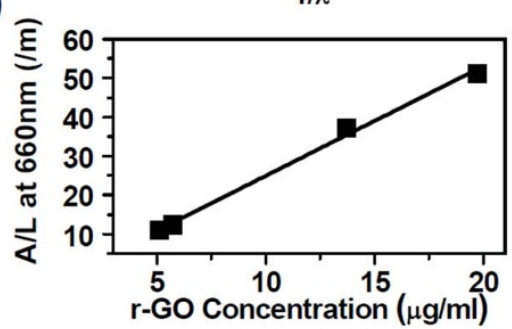

Figure $3 \mid$ (a) UV absorption curve comparing the absorbance vs wavelength ( $\lambda$ ) for different graphene and GA. (b) band-gap curve of G and r-GO. (A/L) vs. graphene concentration for (c) G and d) r-GO. 
Table 1 Summary of extinction coefficient data at $660 \mathrm{~nm}$ compared with other surfactant exfoliated graphene materials

\begin{tabular}{lcc} 
Material & Extinction Coefficient $\left(\mathrm{m}^{*} \mathrm{mg}^{\left.-1 * \mathrm{~m}^{-1}\right)}\right.$ & Additional Sources \\
\hline $\mathrm{G}$ & 5422 & 2813 \\
$\mathrm{r}-\mathrm{GO}$ & 6600 & 20 \\
$\mathrm{G}$ from sonication in solvent & &
\end{tabular}

It is also important to investigate the morphology and size of the two different graphene. From SEM analysis, illustrated in figure 4(a), the $\mathrm{G}$ flakes appear to be rigid, and vary widely in size anywhere from a few hundred nanometers in their longest dimension to around $2 \mu \mathrm{m}$ (measured average $300 \mathrm{~nm}$ wide, $2 \mathrm{um}$ long). The flakes are thin enough that they appear semi-transparent under the SEM but the corners remain sharp and the edges are very well defined. The bright field created by sheets perpendicular to the imaging plane show us that the layers are on the order of only a few nanometers. This morphology closer to the graphene produced through intercalation and thermal shock ${ }^{37,38}$. The flake surface appears to be mostly free of defects based on the very smooth surface texture observed. This is in contrast to the r-GO in Figure 4(b), which displays highly wrinkled morphology with a high degree of out-of-plane bending. The curvature makes it difficult to discern the boundaries between sheets and the ribbon-like edges are full of folds and a high degree of curvature. Thus, graphene produced through our current procedure better preserves the characteristics and properties of pure graphene sheets produced by physical scotch tape peeling ${ }^{39}$.

To further investigate the size and dimension of the $G$ flakes we consider the TEM images in figure 4(c) and (d). The wide-field image in figure 4(c) reveals some flake aggregation and clustering. The small rigid flakes are stacked flat but twisted in random orientations providing a clear distinction between flakes. High resolution TEM of the flake edges reveals the well defined layer structure of the multilayer graphene and figure $4(\mathrm{~d})$ is representative of many TEM images taken which reveal graphene flakes containing on average 7 layers. The r-GO wide field image again reveals the wrinkled sheets inherent to the large number of basal plane defects. In comparison to the $\mathrm{G}$ we can see that the sheets appear larger and less clustered. The high

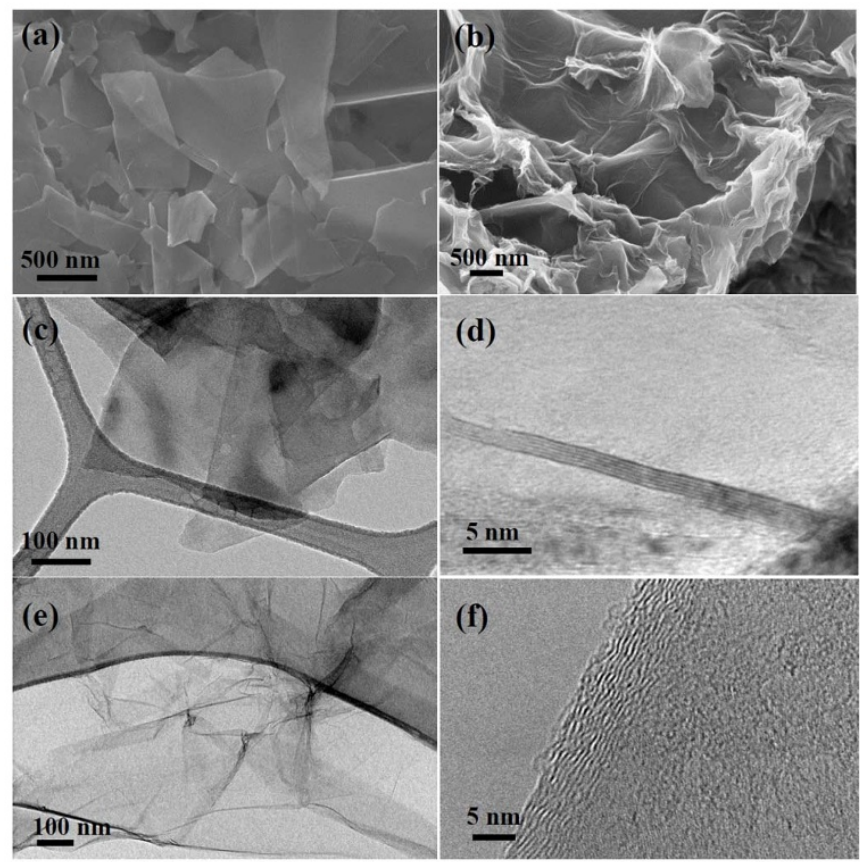

Figure 4 SEM of (a) G and (b) r-GO, as well as, wide field and high resolution TEM images of (c,d) G and (e,f) r-GO. resolution TEM images depict the thin ribbon-like morphology of the $\mathrm{r}-\mathrm{GO}$ also contains an average of 5 layers, equating to a thickness of around $2 \mathrm{~nm}$. TEM observation is in agreement that $\mathrm{G}$ preserves more rigidity than $\mathrm{r}-\mathrm{GO}$, but the flakes are slightly thicker.

Raman spectra in Figure 5 act as a tool for determining relative defect concentrations. In graphite the $\mathrm{D}$-band $\left(\mathrm{I}_{\mathrm{d}}, 1350 \mathrm{~cm}^{-1}\right)$ is negligible compared to the high G-band $\left(\mathrm{I}_{\mathrm{g}}, 1580 \mathrm{~cm}^{-1}\right)$ and a moderately intense $2 \mathrm{D}$ band is visible at higher wave numbers. The $\mathrm{D}$ band represents disorder in the graphitic structure, enabling defect content analysis by comparing the intensity of the two characteristic bands. As expected a large defect ratio $\left(\mathrm{I}_{\mathrm{d}} / \mathrm{I}_{\mathrm{g}}\right)$ of 1.31 is derived for $\mathrm{r}$ GO in figure 5(a), confirming the high concentrations of both basal and edge defects due to the harsh oxidation process. Figure 5(a) also reveals $G$ illustrates a much smaller shift in the defect ratio $(\sim 0.25)$. This low increase in the D-band indicates the mild exfoliation process which leads to very few basal plane defects and only moderate levels of edge defects. As shown in Table 2, the defect density for $G$ is even smaller than that observed for most surfactant based exfoliation methods previously reported. The low number of edge defects for the $G$ flakes further supports the unaltered graphitic character of the basal plane.

Raman analysis has also been shown in literature to be an effective means for determining flake thickness of graphene materials ${ }^{1,40}$. A characteristic shift in the $2 \mathrm{D}$ peak position and shape is indicative of the transition from graphite to monolayer graphene materials. Interestingly in figure $5(\mathrm{~b})$, the $2 \mathrm{D}$ peak present in graphite is completely lost for $\mathrm{r}-\mathrm{GO}$, while G exhibits the expected shift in the peak position and shape. According to literature the peak broadening, 20$30 \mathrm{~cm}^{-1}$ shift, and loss of the $\sim 2670 \mathrm{~cm}^{-1}$ peak shoulder suggest that the $\mathrm{G}$ flakes are composed of a layers between 5-20 layers in thickness ${ }^{1}$. This is in agreement with the estimated thickness distribution suggested by TEM imaging.

To test for the electrical properties of the $G$ the powders were compressed into thin wafers and subjected to 4-probe analysis shown in figure 6(a). The slope of figure 6(a) was used in junction with the measured width and thickness values for each pellet to determine the average conductivity shown in figure 6(b). For comparison, a highly conductive single-walled carbon nanotube (SWNT) film was also tested to reveal an average conductivity of $200 \mathrm{~S} / \mathrm{cm}$, close to the literature published result for SWNTs ${ }^{41}$. The low defect concentration in the graphitic structure of the $G$ pellet enabled us to achieve around $100 \mathrm{~S} / \mathrm{cm}$. Comparable to the $15-72 \mathrm{~S} / \mathrm{cm}$ achieved by other liquid-phase dispersion techniques demonstrated by others previously ${ }^{14,16,20}$. The experimentally measured conductivities of the graphene film is significantly lower than the conductivity of a single graphene sheet, (theoretical in-plane conductivity $\sim 10^{6} \mathrm{~S} / \mathrm{cm}^{42}$ ), which suggests that the resistance of the film is dominated by the resistance of the inter-particle junctions ${ }^{41}$. The conductivity of the $r$ GO pellet was only $5 \mathrm{~S} / \mathrm{cm}$ due to the previously discussed basal plane defects. A literature survey of r-GO conductivity suggests values ranging from $0.05-298 \mathrm{~S} / \mathrm{cm}$ depending on the technique used and degree of reduction ${ }^{1,43,44}$. The r-GO we report is well within this range, but less than the high values reported for annealed graphene films which further reduce the r-GO and minimize interparticle resistances. We find that the $\mathrm{G}$ film produced by the current process has 20 times higher electrical conductivity than that of the $r$ GO film. 

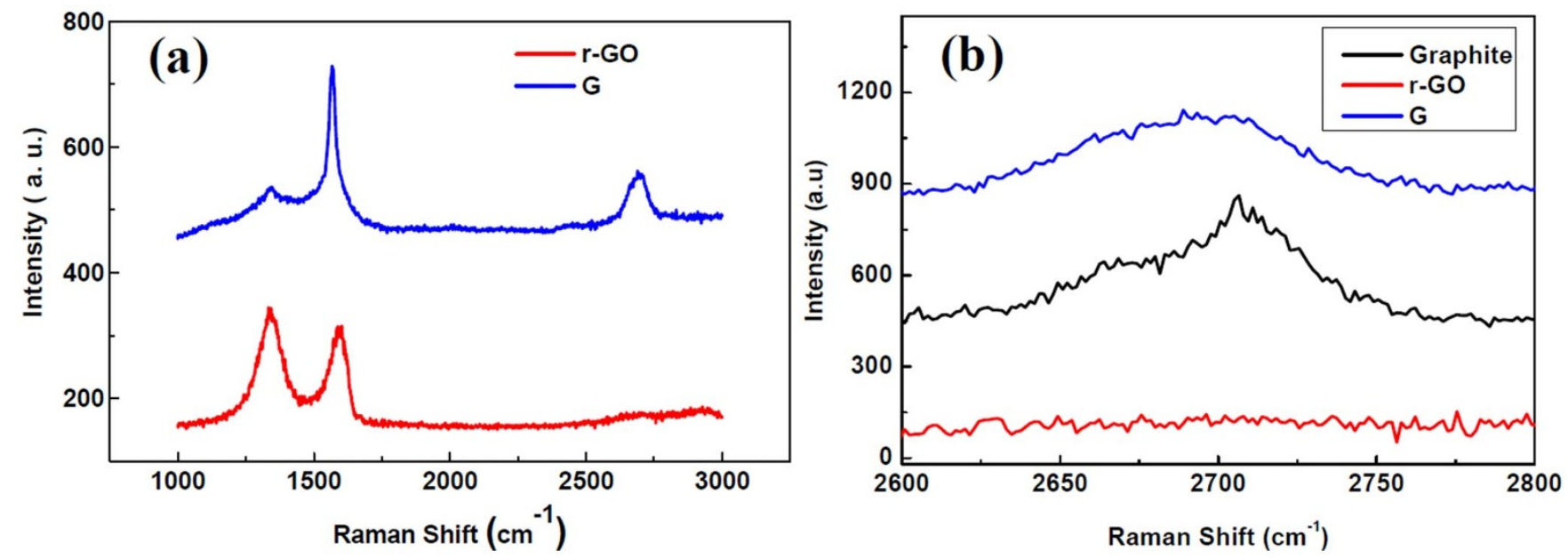

Figure $5 \mid$ (a) Comparison of Raman Spectra between G (blue) and r-GO (red) to determine defect concentration. (b) 2D subsection of spectra with addtional graphite (black) comparison.

\section{Discussion}

The low power sonication technique places stress on the graphite particles by strong sonophysical energy. This stress is transferred throughout the $\mathrm{sp}^{2}$ hybridized carbons in the graphene planes, weakening the attraction between the layers created by the van der waals forces that hold the graphene sheets together. The $10 \mathrm{~nm}$ hydrodynamic radius of GA suggests the polymers are too large to intercalate and overcome the $0.35 \mathrm{~nm}$ spacing of the graphite planes ${ }^{24,25}$. However, the dark black color of the graphene powder, Raman peak shift, and high resolution TEM results strongly support the exfoliation of initial graphite into multi-layer graphene flakes.

We expect the GA likely adsorbs to the exposed surfaces of the graphite, creating a barrier to aggregation and allowing the graphite to slowly exfoliate in the form of undamaged flakes. We believe that GA functions similar to the way surfactant does, but without the formation of micelles. Despite being comparably thin to the r-GO materials, the $\mathrm{G}$ exhibits rigid morphology. This rigidity indicates a comparably low number of basal plane defects. Further, the lower overall defect ratios for the $G$ are much lower than for r-GO. Over time, the sonication stress can also induce scission along the basal plane, reducing the size of the particles, accounting for the edge plane defects that remain and allowing further graphite exfoliation.

The strong adsorption of the GA to the graphene makes separation difficult. Even after multiple high speed centrifugation cycles and extensive washing by filtration the resulting $\mathrm{G}$ were shown to exhibit a significant weight percentage of GA residue anchored to the graphene. In order to overcome this challenge, we have applied a novel strategy to hydrolyze the bonds in the GA structure with acid, leaving the more stable graphene flakes undamaged. This is supported by the low defect ratio observed for G. We have therefore demonstrated that with the assistance of the environmental friendly biopolymer, Gum Arabic, we are able to produce 5-10 layer graphene through mild sonication. Electrical conductivity was almost 20 times higher than
r-GO and comparable to SWNT at high mass loading. The process is simple, scalable, and with high yield (5 wt\%), leads to the possibility for mass production of multi-layer graphene flakes. In addition, the under-exfoliated graphite which is removed before purification suggests in future work we could optimize the initial mass of graphite or recycle the partially exfoliated flakes to further increase the percent yield.

\section{Methods}

Production of graphene with Gum Arabic. Natural graphite powder was purchased from Alfa Aesar (thickness 5-15 $\mu \mathrm{m}$ ) and used without further treatment. Gum Arabic was purchased from Sigma Aldrich. The purified single-walled carbon nanotubes used for electrical conductivity comparison reason were purchased from Carbon Solutions, Inc. GA powder was dissolved in $1 \mathrm{~L}$ of DI water to create a solution from $0.5-5 \mathrm{wt} \%$. Then, $10 \mathrm{~g}$ of graphite was added to form our graphite solution. The graphene dispersion was generated using extended low power ultrasonication bath (Branson 5510) for 100 hours. To prevent overheating and maintain efficiency the water in the sonication bath was changed to maintain the temperature lower than $30^{\circ} \mathrm{C}$. Upon completion the dispersion was left to sit $24 \mathrm{hrs}$ to enable separation of large unstable graphite aggregates. The dispersed GA-G was collected. The sample was further isolated by a low speed centrifugation of $500 \mathrm{rpm}$ for $30 \mathrm{~min}$. The supernatant after filtration and dry, denoted as GA-G, containing graphene particles was kept for testing and for further treatment.

Separation of graphene from Gum Arabic. To totally remove GA, nitric acid treatment was further used on GA-G. The final product was moved to a freeze dryer and the resulting black powder was denoted as $\mathrm{G}$ containing pure graphene with mass yield was up to $0.5 \mathrm{~g}$, or $5 \mathrm{wt} \%$ of the original $10 \mathrm{~g}$ graphite used.

Regular graphene production. For comparison we prepared a chemically reduced graphene using the same graphite precursor oxidized through our previously reported modified Hummers method ${ }^{45-48}$. To reduce the graphene oxide (GO), a solution of $400 \mathrm{mg} \mathrm{GO}$ and $40 \mathrm{~mL}$ DDI water were sonicated and then sodium carbonate was added until $\mathrm{pH}$ reached 10 . Next, $3 \mathrm{~g}$ of sodium borohydride $(98 \%$ min., EMD chemicals) was dissolved in $50 \mathrm{~mL}$ of DDI water before it was added to the reaction vessel and allowed to react at $80^{\circ} \mathrm{C}$ for 48 hours. The resulting graphene powder (r-GO) was filtered and washed with DI water and ethanol before being dried.

Table 2 | Summary of Raman Characterization of the D, $G$ and $2 D$ bands of $G$ and $r-G O$, compared to literature work

\begin{tabular}{lccc} 
Material & $\mathrm{I}_{\mathrm{D}} / \mathrm{I}_{\mathrm{G}}$, Defect Ratio & 2D Peak Analysis $\left(\mathrm{cm}^{-1}\right)$ & Additional Sources \\
\hline $\mathrm{G}$ & 0.25 & Broad, Centered at 2680 \\
r-GO & 1.31 & No Peak \\
Surfactant Exfoliated & $0.3-1.4$ & - & $15,19,20,49-51$ \\
Graphite & $0-0.2$ & Sharp, Centered at 2700-2710 & 15 \\
Single Layer Graphene & - & Shoulder extends to 2650-2660 & 52 \\
5-10 layer Graphene & - & Sharp, Centered at 2650 & 52 \\
\hline
\end{tabular}



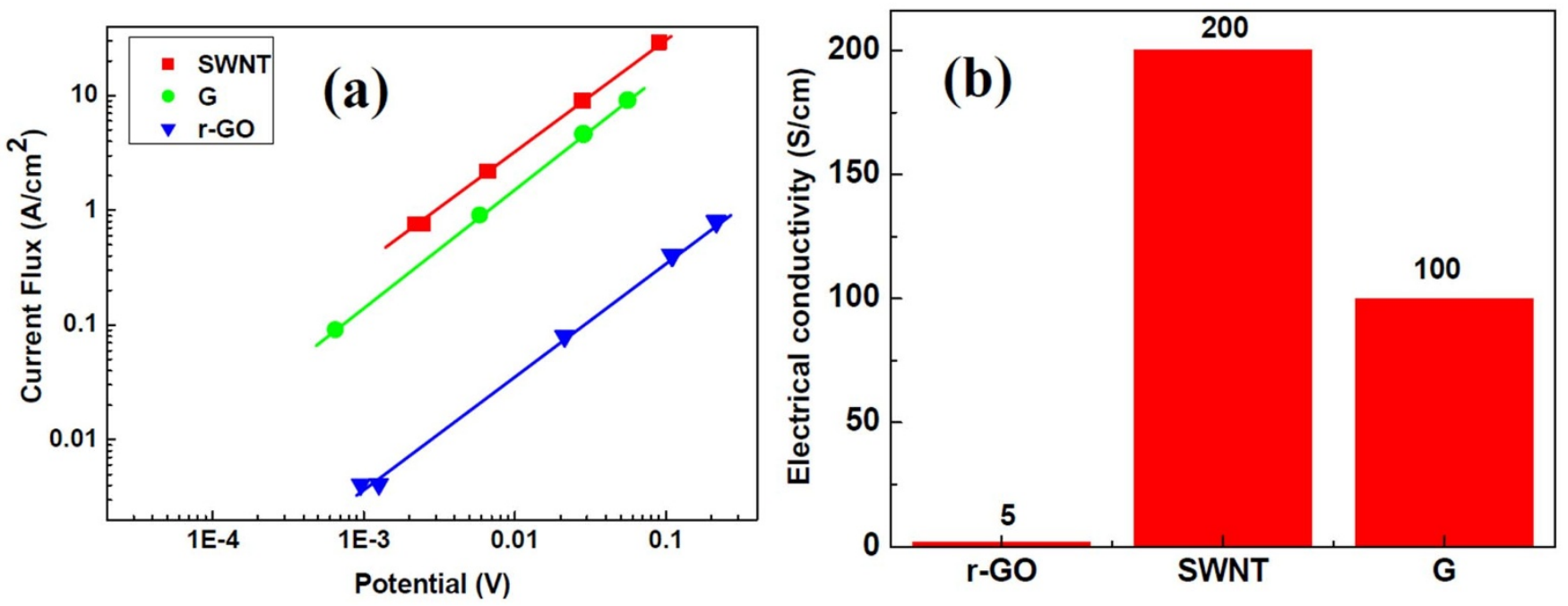

Figure 6 (a) 4-probe electrical conductivity results for G, r-GO, and SWNT, respectively. (b) Average calculated electrical conductivity values for the range tested.

Characterization. Tyndall effect was shown for diluted samples at a concentration of $0.02 \mathrm{mg} / \mathrm{mL}$, using a consumer grade laser pointer. Optical absorption measurements were taken with a Genesys 10 UV spectrometer. Absorption measurements were used to estimate residual GA levels. TGA was performed to estimate graphitic content using a slow temperature ramp of $5^{\circ} \mathrm{C} \cdot \mathrm{min}^{-1}$ up to a temperature of $900^{\circ} \mathrm{C}$, under $50 \mathrm{~cm}^{3} / \mathrm{min}$ flow rate of air. SEM characterization of material morphology was prepared by loading a few milligram of freeze dried sample onto carbon tape. TEM was prepared by re-dispersing a small quantity of dry sample and dropping a few milliliters onto a carbon grid. RAMAN (633 nm laser) was used to analyze edge defects and estimate layer thickness. Electrical conductivity was determined by pressing the graphene and SWNT powder materials at 4000 psi to form small pellets that approach the maximum density of the material and a custom 4-probe electrochemical test setup with $5 \mathrm{~mm}$ spacing was used for measurement.

1. Singh, V. et al. Graphene based materials: past, present and future. Progress in Materials Science 56, 1178-1271 (2011).

2. Rao, C. N. R., Sood, A. K., Subrahmanyam, K. S. \& Govindaraj, A. Graphene: the new two-dimensional nanomaterial. Angew. Chem. Int. Ed. 48, $7752-7777$ (2009).

3. Deheer, W. et al. Epitaxial graphene. Solid State Comm. 143, 92-100 (2007).

4. Yan, Z. et al. Growth of bilayer graphene on insulating substrates. ACS nano 5, 8187-8192 (2011)

5. Chen, C. et al. Self-assembled free-standing graphite oxide membrane. Adv. Mater. 21, 3007-3011 (2009).

6. Geng, D. et al. Nitrogen doping effects on the structure of graphene. Applied Surface Science 257, 9193-9198 (2011).

7. Zhou, X. \& Liu, Z. A scalable, solution-phase processing route to graphene oxide and graphene ultralarge sheets. Chem. Commun. 46, 2611-2613 (2010).

8. Park, S. \& Ruoff, R. S. Chemical methods for the production of graphenes. Nat. Nano. 4, 217-224 (2009).

9. Tung, V. C., Allen, M. J., Yang, Y. \& Kaner, R. B. High-throughput solution processing of large-scale graphene. Nat. Nano. 4, 25-29 (2009).

10. Becerril, H. A. et al. Evaluation of solution-processed reduced graphene oxide films as transparent conductors. ACS nano 2, 463-470 (2008).

11. Shin, H.-J. et al. Efficient reduction of graphite oxide by sodium borohydride and its effect on electrical conductance. Adv. Funct. Mater. 19, 1987-1992 (2009).

12. Stankovich, S. et al. Synthesis of graphene-based nanosheets via chemical reduction of exfoliated graphite oxide. Carbon 45, 1558-1565 (2007).

13. Eda, G., Fanchini, G. \& Chhowalla, M. Large-area ultrathin films of reduced graphene oxide as a transparent and flexible electronic material. Nat. Nano. 3, 270-274 (2008).

14. Hamilton, C. E., Lomeda, J. R., Sun, Z., Tour, J. M. \& Barron, A. R. High-yield organic dispersions of unfunctionalized graphene. Nano Lett. 9, 3460-3462 (2009)

15. Khan, U., O’Neill, A., Lotya, M., De, S. \& Coleman, J. N. High-concentration solvent exfoliation of graphene. Small 6, 864-871 (2010).

16. Hernandez, Y. et al. High-yield production of graphene by liquid-phase exfoliation of graphite. Nat. Nano. 3, 563-568 (2008).

17. Bourlinos, A. B., Georgakilas, V., Zboril, R., Steriotis, T. A. \& Stubos, A. K. Liquidphase exfoliation of graphite towards solubilized graphenes. Small 5, 1841-1845 (2009).

18. Vadukumpully, S., Paul, J. \& Valiyaveettil, S. Cationic surfactant mediated exfoliation of graphite into graphene flakes. Carbon 47, 3288-3294 (2009).
19. Lotya, M. et al. Liquid phase production of graphene by exfoliation of graphite in surfactant/water solutions. J. Am. Chem. Soc. 131, 3611-3620 (2009).

20. Lotya, M., King, P. J., Khan, U., De, S. \& Coleman, J. N. High-concentration, surfactant-stabilized graphene dispersions. ACS nano 4, 3155-3162 (2010).

21. Cserháti, T., Forgács, E. \& Oros, G. Biological activity and environmental impact of anionic surfactants. Environment International 28, 337-348 (2002).

22. Zoller, U. Water reuse/recycling and reclamation in semiarid zones: the israeli case of salination and hard surfactants pollution of aquifers. J. Environ. Eng. 132, 683-688 (2006).

23. Marcomini, A., Pojana, G., Sfriso, A. \& Alonso, J.-M. Q. Behaviour of anionic and nonionic surfactant and their persistent metabolites in the venice lagoon, Italy. Environ. Toxicol. Chem. 19, 2000-2007 (2000).

24. Swenson, H. A., Kaustinen, H. M., Kaustinen, O. A. \& Thompson, N. S. Structure of gum arabic and its configuration in solution. J. Polym. Sci. A-2 Polym. Phys. 6, 1593-1606 (1968).

25. Cozic, C., Picton, L., Garda, M.-R., Marlhoux, F. \& Le Cerf, D. Analysis of arabic gum: Study of degradation and water desorption processes. Food Hydrocolloids 23, 1930-1934 (2009).

26. Bandyopadhyaya, R., Nativ-roth, E., Regev, O., Yerushalmi-rozen, R. \& Sheva, B. Stabilization of individual carbon nanotubes in aqueous solutions. Nano Lett. 2, 25-28 (2002).

27. Dror, Y., Cohen, Y. \& Yerushalmi-rozen, R. Structure of gum arabic in aqueous solution. J. Polym. Sci. B Polym. Phys. 44, 3265-3271 (2006).

28. Erni, P. et al. Interfacial rheology of surface-active biopolymers: acacia senegal gum versus hydrophobically modified starch. Biomacromolecules 8, 3458-3466 (2007).

29. Choi, E.-K. et al. High-yield exfoliation of three-dimensional graphite into twodimensional graphene-like sheets. Chemical Commun. 46, 6320-6322 (2010).

30. Tauc, J., Grigorovici, R. \& Vancu, A. Optical properties and electronic structure of amorphous germanium. Phys. Status Solidi B 15, 627-637 (1966).

31. Tauc, J. Electronic properties of amorphous materials. Science 158, 1543-1548 (1967).

32. Ci, L. et al. Atomic layers of hybridized boron nitride and graphene domains. Nat. Mater. 9, 430-435 (2010).

33. Mathkar, A. et al. Controlled, stepwise reduction and band gap manipulation of graphene oxide. J. Phys. Chem. Lett. 3, 986-991 (2012).

34. Wei, J. et al. Preparation of highly oxidized nitrogen-doped carbon nanotubes. Nanotechnology 23, 155601 (2012).

35. Zhao, B. et al. Extinction coefficients and purity of single-walled carbon nanotubes. J. Nanosci. Nanotech. 4, 995-1004 (2004).

36. Zhao, B. et al. Study of the extinction coefficients of single-walled carbon nanotubes and related carbon materials. J. Phys. Chem. B 108, 8136-8141 (2004).

37. Yu, A. et al. Enhanced thermal conductivity in a hybrid graphite nanoplatelet carbon nanotube filler for epoxy composites. Adv. Mater. 20, 4740-4744 (2008).

38. Yu, A., Ramesh, P., Itkis, M. E., Bekyarova, E. \& Haddon, R. C. Graphite nanoplatelet-epoxy composite thermal interface materials. J. Phys. Chem. C 111, 7565-7569 (2007).

39. Allen, M. J., Tung, V. C. \& Kaner, R. B. Honeycomb carbon: a review of graphene. Chemical Reviews 110, 132-145 (2010).

40. Chen, Z. et al. Three-dimensional flexible and conductive interconnected graphene networks grown by chemical vapour deposition. Nat. Mater. 10, 424-428 (2011)

41. Bekyarova, E. et al. Electronic properties of single-walled carbon nanotube networks. J. Am. Chem. Soc. 127, 5990-5995 (2005). 
42. Chen, J.-H., Jang, C., Xiao, S., Ishigami, M. \& Fuhrer, M. S. Intrinsic and extrinsic performance limits of graphene devices on $\mathrm{SiO}_{2}$. Nat. Nano. 3, 206-209 (2008).

43. Zhao, X. et al. Low-cost preparation of a conductive and catalytic graphene film from chemical reduction with $\mathrm{AlI}_{3}$. Carbon 50, 3497-3502 (2012).

44. Li, D., Muller, M. B., Gilje, S., Kaner, R. B. \& Wallace, G. G. Processable aqueous dispersions of graphene nanosheets. Nat Nano 3, 101-105 (2008).

45. Hummers, W. Preparation of graphitic oxide. J. Am. Chem. Soc. 80, 1339 (1958).

46. Davies, A. et al. Graphene-based flexible supercapacitors: pulseelectropolymerization of polypyrrole on free-standing graphene films. J. Phys. Chem. C 115, 17612-17620 (2011).

47. Yu, A., Roes, I., Davies, A. \& Chen, Z. Ultrathin, transparent, and flexible graphene films for supercapacitor application. App. Phys. Lett. 96, 253105 (2010).

48. Yu, A., Sy, A. \& Davies, A. Graphene nanoplatelets supported $\mathrm{MnO}_{2}$ nanoparticles for electrochemical supercapacitor. Synthetic Metals 161, 2049-2054 (2011).

49. Smith, R. J., King, P. J., Wirtz, C., Duesberg, G. S. \& Coleman, J. N. Lateral size selection of surfactant-stabilised graphene flakes using size exclusion chromatography. Chemical Physics Letters 531, 169-172 (2012).

50. Seo, J., Green, A., Antaris, A. \& Hersam, M. High-concentration aqueous dispersions of graphene using nonionic biocompatible block copolymers. J. Phys. Chem. Lett. 2, 1004-1008 (2011).

51. Green, A. \& Hersam, M. C. Solution phase production of graphene with controlled thickness via density differentiation. Nano Lett. 9, 4031-4036 (2009).

52. Ferrari, A. C. et al. Raman spectrum of graphene and graphene layers. Phys. Rev. Lett. 97, 187401 (2006)

\section{Acknowledgments}

This research was financially supported by the Natural Sciences and Engineering Research Council of Canada (NSERC) as well as the University of Waterloo.

\section{Author contributions}

V.C., C.T. and A.Y. designed the experiments and wrote the manuscript text. V.C., B.K. and B.S. performed synthesis and purification of the materials as well as conductivity characterization. V.C. performed the remaining characterization. All authors reviewed the manuscript.

\section{Additional information}

Competing financial interests: The authors declare no competing financial interests.

License: This work is licensed under a Creative Commons

Attribution-NonCommercial-NoDerivs 3.0 Unported License. To view a copy of this license, visit http://creativecommons.org/licenses/by-nc-nd/3.0/

How to cite this article: Chabot, V., Kim, B., Sloper, B., Tzoganakis, C. \& Yu, A. High yield production and purification of few layer graphene by Gum Arabic assisted physical sonication. Sci. Rep. 3, 1378; DOI:10.1038/srep01378 (2013). 